Document downloaded from:

http://hdl.handle.net/10251/169537

This paper must be cited as:

Bermúdez, T.; Bonilla, A.; Muller, V.; Peris Manguillot, A. (2020). Cesaro bounded operators in Banach spaces. Journal d Analyse Mathématique. 140(1):187-206.

https://doi.org/10.1007/s11854-020-0085-8

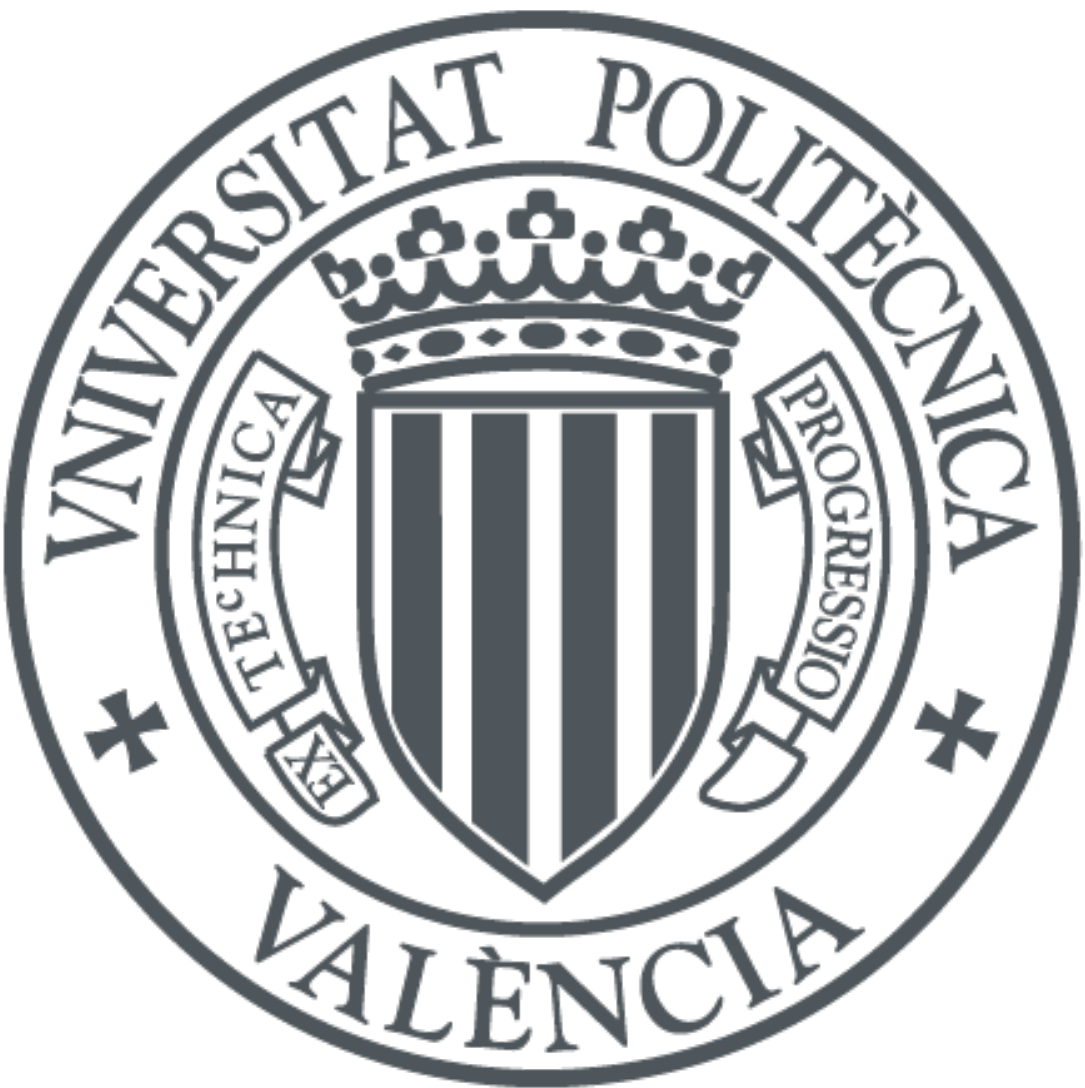

The final publication is available at

https://doi.org/10.1007/s11854-020-0085-8

Copyright Springer-Verlag

Additional Information 


\title{
Cesàro bounded operators in Banach spaces
}

\author{
T. Bermúdez, A. Bonilla, V. Müller and A. Peris *
}

February 9, 2018

\begin{abstract}
We study several notions of boundedness for operators. It is known that any power bounded operator is absolutely Cesàro bounded and strongly Kreiss bounded (in particular, uniformly Kreiss bounded). The converses do not hold in general. In this note, we give examples of topologically mixing (hence, not power bounded) absolutely Cesàro bounded operators on $\ell^{p}(\mathbb{N}), 1 \leq p<\infty$, and provide examples of uniformly Kreiss bounded operators which are not absolutely Cesàro bounded. These results complement a few known examples (see [26] and [2]). We also obtain a characterization of power bounded operators which generalizes a result of Van Casteren [31]. In [2] Aleman and Suciu asked if every uniformly Kreiss bounded operator $T$ on a Banach space satisfies that $\lim _{n \rightarrow \infty}\left\|\frac{T^{n}}{n}\right\|=0$. We solve this question for Hilbert space operators and, moreover, we prove that, if $T$ is absolutely Cesàro bounded on a Banach (Hilbert) space, then $\left\|T^{n}\right\|=o(n)\left(\left\|T^{n}\right\|=o\left(n^{\frac{1}{2}}\right)\right.$, respectively). As a consequence, every absolutely Cesàro bounded operator on a reflexive Banach space is mean ergodic.
\end{abstract}

\section{Introduction}

Throughout this article $X$ stands for a Banach space, the symbol $B(X)$ denotes the space of bounded linear operators defined on $X$, and $X^{*}$ is the space of continuous linear functionals on $X$.

Given $T \in B(X)$, we denote the Cesàro mean by

$$
M_{n}(T) x:=\frac{1}{n+1} \sum_{k=0}^{n} T^{k} x
$$

for all $x \in X$.

We need to recall some definitions concerning the behaviour of the sequence of Cesàro means $\left(M_{n}(T)\right)_{n \in \mathbb{N}}$.

${ }^{*}$ The first, second and four authors were supported by MINECO and FEDER, Project MTM2016-75963-P. The third author was supported by grant No. 17-27844S of GA CR and RVO: 67985840. The fourth author was also supported by Generalitat Valenciana, Project PROMETEO/2017/102. 
Definition 1.1. A linear operator $T$ on a Banach space $X$ is called

1. Uniformly ergodic if $M_{n}(T)$ converges uniformly.

2. Mean ergodic if $M_{n}(T)$ converges in the strong operator topology of $X$.

3. Weakly ergodic if $M_{n}(T)$ converges in the weak operator topology of $X$.

4. Absolutely Cesàro bounded if there exists a constant $C>0$ such that

$$
\sup _{N \in \mathbb{N}} \frac{1}{N} \sum_{j=1}^{N}\left\|T^{j} x\right\| \leq C\|x\|,
$$

for all $x \in X$.

5. Cesàro bounded if the sequence $\left(M_{n}(T)\right)_{n \in \mathbb{N}}$ is bounded.

An operator $T$ is said to be power bounded if there is a $C>0$ such that $\left\|T^{n}\right\|<C$ for all $n$.

The class of absolutely Cesàro bounded operators was introduced by Hou and Luo in [15]. For power-bounded operators, weak ergodicity is equivalent to mean ergodicity (see [19, Theorem II.1.1]). There exist weakly ergodic operators on Hilbert spaces which are not mean ergodic (hence not power-bounded), see [10, page 454] and [30, Example 3.1].

The first example of a mean ergodic operator which is not power-bounded was given by Hille ([16], where $\left.\left\|T^{n}\right\| \sim n^{1 / 4}\right)$. An example of a mean ergodic operator $T$ on $L^{1}(\mathbb{Z})$ with $\lim \sup _{n}\left\|T^{n}\right\| / n>0$ was obtained in [18] (Certainly, $\left\|T^{n} x\right\| / n \rightarrow 0$ for every $x \in L^{1}(\mathbb{Z})$ ).

Van Casteren [31, page 61] defined $T$ on a Banach space $X$ to be mean square bounded if for some constant $M$

$$
\sup _{N \in \mathbb{N}} \frac{1}{N} \sum_{j=1}^{N}\left\|T^{j} x\right\|^{2} \leq M^{2}\|x\|^{2},
$$

for all $x \in X$. Van Casteren proved that if $T$ and $T^{*}$ on a Hilbert space are mean square bounded, then $T$ is power-bounded. Mean square boundedness lies between power-boundedness and absolute Cesàro boundedness, by Cauchy- Schwarz inequality, which leads to the question whether absolute Cesàro boundedness implies mean square boundedness. We give the negative answer to this question.

Additional results about mean ergodicity of operators on spaces of analytic functions can be found in $[5,6]$.

Definition 1.2. For an operator $T$ we have three notions of Kreiss boundedness, ordered by strength, if there exists $C>0$ such that 
1. Strongly Kreiss bounded:

$$
\left\|(\lambda I-T)^{-k}\right\| \leq \frac{C}{(|\lambda|-1)^{k}} \quad \text { for all }|\lambda|>1 \text { and } k=1,2, \cdots
$$

2. Uniformly Kreiss bounded:

$$
\left\|\sum_{k=0}^{n} \lambda^{-k-1} T^{k}\right\| \leq \frac{C}{|\lambda|-1} \text { for all }|\lambda|>1 \text { and } n=0,1,2, \cdots
$$

3. Kreiss bounded:

$$
\left\|(\lambda I-T)^{-1}\right\| \leq \frac{C}{|\lambda|-1} \quad \text { for all }|\lambda|>1 .
$$

Remark 1.1. 1. In [22, Corollary 3.2], it is proved that an operator $T$ is uniformly Kreiss bounded if and only if there is a $C$ such that

$$
\left\|M_{n}(\lambda T)\right\| \leq C \text { for }|\lambda|=1 \text { and } n=0,1,2, \cdots .
$$

2. We recall [13] that $T$ is strongly Kreiss bounded if and only if

$$
\left\|e^{z T}\right\| \leq M e^{|z|}, \text { for all } z \in \mathbb{C} .
$$

3. In [13], it is shown that every strong Kreiss bounded operator is uniformly Kreiss bounded. It was shown in [22, Section 5] that uniform Kreiss boundedness does not imply strong Kreiss boundedness. McCarthy (see [21], [26]) proved that if $T$ is strong Kreiss bounded then $\left\|T^{n}\right\| \leq C n^{\frac{1}{2}}$ (see also [20, Theorem 2.1]). McCarthy also produced an example of a strong Kreiss bounded operator which is not power bounded.

4. If $T$ is Kreiss bounded, then $\left\|T^{n}\right\| \leq C n$ [20, formula (2.4)]. By Nevanlinna [24, Theorem 6], there are Kreiss bounded operators $T$ on Banach spaces with $\left\|T^{n}\right\| \geq C n$ for some $C>0$. There exist Kreiss bounded operators which are not Cesàro bounded, and conversely [29].

5. On finite-dimensional Hilbert spaces, the classes of uniformly Kreiss bounded, strong Kreiss bounded, Kreiss bounded and power bounded operators are equal.

6. By (1) any absolutely Cesàro bounded operator is uniformly Kreiss bounded.

Let $X$ be the space of all bounded analytic functions $f$ on the unit disk of the complex plane such that their derivatives $f^{\prime}$ belong to the Hardy space $H^{1}$, endowed with the norm

$$
\|f\|=\|f\|_{\infty}+\|f\|_{H^{1}} .
$$


Then the multiplication operator, $M_{z}$, acting on $X$ is Kreiss bounded but it fails to be power bounded. Moreover, this operator is not uniformly Kreiss bounded (see $[27])$.

Furthermore, for the Volterra operator $V$ acting on $L^{p}[0,1], 1 \leq p \leq \infty$, we have that $I-V$ is uniformly Kreiss bounded, for $p=2$ it is power bounded (see [22]), and it is asked if every uniformly Kreiss bounded operator on a Hilbert space is power bounded. This is related to the following question in [2, page 279] (see also, [28]):

Question 1.1. If $T$ is a uniformly Kreiss bounded operator on a Banach space, does it follow that $\lim _{n \rightarrow \infty} \frac{\left\|T^{n}\right\|}{n}=0$ ?

Graphically, we show the implications between the above definitions.

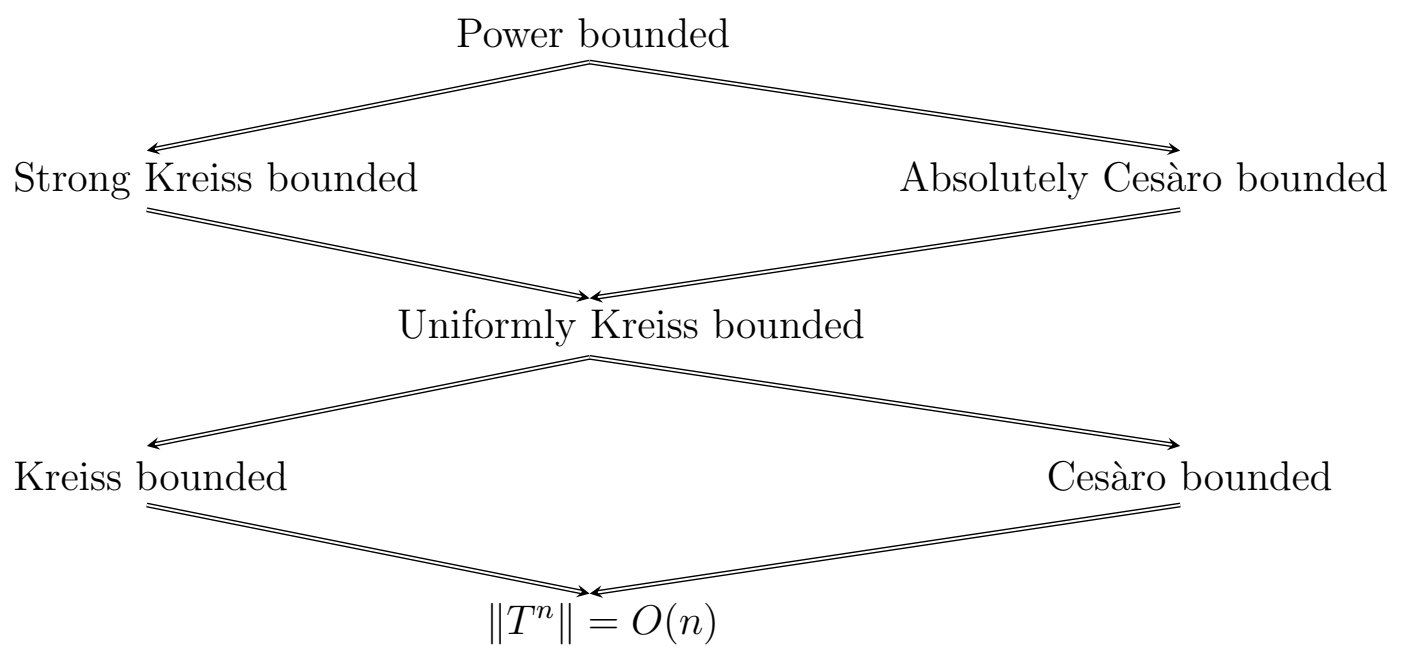

Figure 1: Implications among different definitions related with Kreiss bounded and Cesàro bounded operators in Banach spaces.

We recall the following definition that allow us to study some properties of orbits related to the behavior of the sequence $\left(M_{n}(T)\right)_{n \in \mathbb{N}}$.

Definition 1.3. Let $T \in B(X)$. $T$ is topologically mixing if for any pair $U, V$ of non-empty open subsets of $X$, there exists some $n_{0} \in \mathbb{N}$ such that $T^{n}(U) \cap V \neq \emptyset$ for all $n \geq n_{0}$.

Examples of absolutely Cesàro bounded mixing operators on $\ell^{1}(\mathbb{N})$ can be found in [15], and in [7] (see also [8]).

The paper is organized as follows: We prove the optimal asymptotic behavior of $\left\|T^{n}\right\|$ for absolutely Cesàro bounded operators and for uniformly Kreiss bounded operators. In particular, we show that, for any $0<\varepsilon<1$, there exists an absolutely Cesàro bounded mixing operator $T$ on $\ell^{p}(\mathbb{N}), 1 \leq p<\infty$, with $\left\|T^{n}\right\|=(n+1)^{\frac{1-\varepsilon}{p}}$. We also prove that power boundedness of $T$ is equivalent to the fact that $T$ and $T^{*}$ 
are mean square bounded operators, and to the fact that $T$ and $T^{*}$ are absolutely Cesàro bounded. Moreover, we show that any absolutely Cesàro bounded operator on a Banach space, and any uniformly Kreiss bounded operator on a Hilbert space, satisfies $\left\|T^{n}\right\|=o(n)$. For absolutely Cesàro bounded operators $T$ on Hilbert spaces we get $\left\|T^{n}\right\|=o\left(n^{\frac{1}{2}}\right)$.

\section{Absolutely Cesàro bounded operators}

It is immediate that any power bounded operator is absolutely Cesàro bounded. In general, the converse is not true.

By $e_{n}, n \in \mathbb{N}$, we denote the standard canonical basis, $e_{n}=\left(\delta_{n k}\right)_{k \in \mathbb{N}}:=$ $(\underbrace{0, \ldots, 0}_{n-1}, 1,0, \ldots)$, in $\ell^{p}(\mathbb{N})$ for $1 \leq p<\infty$.

The following theorem yields a variety of absolutely Cesàro bounded operators with different behavior on $\ell^{p}(\mathbb{N})$.

Theorem 2.1. Let $T$ be the unilateral weighted backward shift on $\ell^{p}(\mathbb{N})$ with $1 \leq$ $p<\infty$ defined by $T e_{1}:=0$ and $T e_{k}:=w_{k} e_{k-1}$ for $k>1$. If $w_{k}:=\left(\frac{k}{k-1}\right)^{\bar{\alpha}}$ with $0<\alpha<\frac{1}{p}$, then $T$ is absolutely Cesàro bounded on $\ell^{p}(\mathbb{N})$ and it is not power bounded.

Proof. By definition, $\left\|T^{n} e_{k}\right\|=\left(\frac{k}{k-n}\right)^{\alpha}$ for $k>n$, so

$$
\left\|T^{n}\right\| \geq\left\|T^{n} e_{n+1}\right\|=(n+1)^{\alpha} ;
$$

hence $T$ is not power bounded.

Denote $\varepsilon:=1-\alpha p$. Then $\varepsilon>0$ and $\alpha=\frac{1-\varepsilon}{p}$. Fix $x \in \ell^{p}(\mathbb{N})$ with $\|x\|=1$ given by $x:=\sum_{j=1}^{\infty} \alpha_{j} e_{j}$ and $N \in \mathbb{N}$. Then

$$
\begin{aligned}
\sum_{n=1}^{N}\left\|T^{n} x\right\|_{p}^{p} & =\sum_{n=1}^{N} \sum_{j=n+1}^{\infty}\left|\alpha_{j}\right|^{p}\left(\frac{j}{j-n}\right)^{1-\varepsilon} \\
& =\sum_{j=2}^{\infty}\left|\alpha_{j}\right|^{p} j^{1-\varepsilon} \sum_{n=1}^{\min \{N, j-1\}}(j-n)^{\varepsilon-1} \\
& =\sum_{j=2}^{2 N}\left|\alpha_{j}\right|^{p} j^{1-\varepsilon} \sum_{n=1}^{\min \{N, j-1\}}(j-n)^{\varepsilon-1}+\sum_{j=2 N+1}^{\infty}\left|\alpha_{j}\right|^{p} \sum_{n=1}^{N}\left(\frac{j}{j-n}\right)^{1-\varepsilon} \\
& \leq \sum_{j=2}^{2 N}\left|\alpha_{j}\right|^{p} j^{1-\varepsilon} \sum_{n=1}^{j-1}(j-1)^{\varepsilon-1}+\sum_{j=2 N+1}^{\infty}\left|\alpha_{j}\right|^{p} \sum_{n=1}^{N}\left(\frac{j}{j-n}\right)^{1-\varepsilon} .
\end{aligned}
$$

Notice that for $j>2 N$ and $n \leq N$, we have that

$$
\left(\frac{j}{j-n}\right)^{1-\varepsilon} \leq 2^{1-\varepsilon}<2 \text {. }
$$


Hence

$$
\sum_{j=2 N+1}^{\infty}\left|\alpha_{j}\right|^{p} \sum_{n=1}^{N}\left(\frac{j}{j-n}\right)^{1-\varepsilon}<2 N \sum_{j=2 N+1}^{\infty}\left|\alpha_{j}\right|^{p} \leq 2 N .
$$

We can estimate the first term of (3) in the following way:

$$
\begin{aligned}
\sum_{n=1}^{j-1}(j-n)^{\varepsilon-1} & =\sum_{n=1}^{j-1} n^{\varepsilon-1}<1+\int_{1}^{j-1} t^{\varepsilon-1} d t \\
& \leq \frac{(j-1)^{\varepsilon}}{\varepsilon}<\frac{j^{\varepsilon}}{\varepsilon} .
\end{aligned}
$$

Thus

$$
\begin{aligned}
\sum_{n=1}^{N}\left\|T^{n} x\right\|_{p}^{p} & \leq \sum_{j=2}^{2 N}\left|\alpha_{j}\right|^{p} j^{1-\varepsilon} \frac{j^{\varepsilon}}{\varepsilon}+\sum_{j=2 N+1}^{\infty}\left|\alpha_{j}\right|^{p} 2 N \\
& =\sum_{j=2}^{2 N}\left|\alpha_{j}\right|^{p} \frac{j}{\varepsilon}+2 N \sum_{j=2 N+1}^{\infty}\left|\alpha_{j}\right|^{p} \\
& \leq \frac{2 N}{\varepsilon} \sum_{j=2}^{2 N}\left|\alpha_{j}\right|^{p}+2 N \sum_{j=2 N+1}^{\infty}\left|\alpha_{j}\right|^{p} \\
& \leq 2 N\left(\frac{1}{\varepsilon}+1\right) .
\end{aligned}
$$

By Jensen's inequality

$$
\left(\frac{1}{N} \sum_{n=1}^{N}\left\|T^{n} x\right\|_{p}\right)^{p} \leq \frac{1}{N} \sum_{n=1}^{N}\left\|T^{n} x\right\|_{p}^{p} \leq 2\left(\frac{1}{\varepsilon}+1\right),
$$

which yields the result.

Remark 2.1. (A) In [11] Derriennic and Lin gave an example of positive Cesàro bounded operator $T$ which is not power bounded on $L^{1}$ (of a countable space). By positivity $\left|T^{n} f\right| \leq T^{n}|f|$, so

$$
\frac{1}{N} \sum_{j=1}^{N}\left\|T^{j} f\right\|_{1} \leq \frac{1}{N} \sum_{j=1}^{N}\left\|T^{j}|f|\right\|_{1}=\left\|\frac{1}{N} \sum_{j=1}^{N} T^{j}|f|\right\|_{1}
$$

by the additivity of the $L^{1}$-norm on positive functions, which shows that $T$ is absolutely Cesàro bounded and it is not power-bounded.

(B) Bonet observed in [9] that any mixing operator $T$ on a Banach space $X$ satisfies $\left\|\left(T^{*}\right)^{n} x\right\| \rightarrow \infty$ for every $x \neq 0$. In particular, if $T$ is mixing then $T^{*}$ cannot be absolutely Cesàro bounded. 
Corollary 2.1. There exist mean square bounded operators on $\ell^{p}(\mathbb{N}), 2 \leq p<\infty$, which are not power bounded.

Proof. It is an immediate consequence of the proof of the Theorem 2.1 when $2 \leq$ $p<\infty$, since we can use the monotonicity of $L^{p}$ norms in probability spaces [25] to obtain:

$$
\left(\frac{1}{N} \sum_{j=1}^{N}\left\|T^{j} f\right\|_{p}^{2}\right)^{1 / 2} \leq\left(\frac{1}{N} \sum_{j=1}^{N}\left\|T^{j} f\right\|_{p}^{p}\right)^{1 / p} \leq(2(\varepsilon+1) / \varepsilon)^{1 / p}
$$

so for $p \geq 2$, the operator $T$ of Theorem 2.1 on $\ell^{p}(\mathbb{N})$ is mean square bounded.

In [17] Kornfeld and Kosek constructed for every $\delta \in(0,1)$ a positive mean ergodic operator $T$ on $L^{1}$ with $\left\|T^{n}\right\| \sim n^{1-\delta}$. By positivity, $T$ is absolutely Cesàro bounded, so $T$ is not strongly Kreiss bounded when $\delta<1 / 2$. The following corollary gives examples in reflexive spaces.

Corollary 2.2. For $1<p<2$, there exist absolutely Cesàro bounded operators on $\ell^{p}(\mathbb{N})$ which are not strongly Kreiss bounded.

Proof. In view of [26, Remark 3], if $T$ is a strong Kreiss bounded operator then $\left\|T^{n}\right\| \leq C n^{\frac{1}{2}}$. The conclusion follows by taking $\frac{1}{2}<\alpha<\frac{1}{p}$ in Theorem 2.1 by $(2)$.

Corollary 2.3. Let $1 \leq p<\infty$ and $0<\varepsilon<1$. Then there exists an absolutely Cesàro bounded operator $T$ on $\ell^{p}(\mathbb{N})$ which is mixing and $\left\|T^{n}\right\|=(n+1)^{\frac{1-\varepsilon}{p}}$ for all $n \in \mathbb{N}$.

Proof. By the begining of the proof of Theorem 2.1 we have that $T$ is absolutely Cesàro bounded and

$$
\left\|T^{n}\right\|=(n+1)^{\frac{1-\varepsilon}{p}} .
$$

Moreover by [14, Theorem 4.8] we have that $T$ is mixing if $\left(\prod_{k=1}^{n} w_{k}\right)^{-1} \rightarrow 0$ as $n \rightarrow \infty$. Indeed

$$
\left(\prod_{k=1}^{n} w_{k}\right)^{-1}=\frac{1}{n^{\alpha}} \rightarrow 0
$$

hence $T$ is mixing.

When $2 \leq p<\infty$, the operator $T$ of Theorem 2.1 is even mean square bounded. It is natural to ask if it is it possible to find examples of absolutely Cesàro bounded operators $T$ which are not mean square bounded. We provide an answer to this question.

Example 2.1. There exist absolutely Cesàro bounded operators which are not mean square bounded. 
Proof. Let $1<p<2$ and $\frac{1}{2}<\alpha<\frac{1}{p}$. Let $T$ be the operator constructed in Theorem 2.1.

Set $n_{k}=2^{k} \quad(k=1,2, \ldots)$. Let $x=\sum_{k=1}^{\infty} k^{-2} e_{n_{k}+1}$. We have

$$
\|x\|_{p}^{p}=\sum_{k=1}^{\infty} k^{-2 p}<\infty .
$$

So $x \in \ell^{p}(\mathbb{N})$.

For each $k$ we have

$$
\frac{1}{n_{k}} \sum_{n=1}^{n_{k}}\left\|T^{n} x\right\|_{p}^{2} \geq \frac{1}{n_{k}}\left\|T^{n_{k}}\left(k^{-2} e_{n_{k}+1}\right)\right\|_{p}^{2} \geq \frac{1}{n_{k}} k^{-4} n_{k}^{2 \alpha}=2^{k(2 \alpha-1)} \cdot k^{-4} \rightarrow \infty
$$

as $k \rightarrow \infty$. So $T$ is not mean square bounded. By Theorem 2.1, $T$ is absolutely Cesàro bounded.

Van Casteren proved that if $T$ and $T^{*}$ on a Hilbert space are mean square bounded, then $T$ is power-bounded [31, Proposition 2.1]. In the following theorem we obtain a complete characterization of power boundedness in terms of the mean square bounded property, and in terms of the absolutely Cesàro bounded property, for operators on general Banach spaces.

Theorem 2.2. Let $T$ be an operator on a Banach space $X$. The following statements are equivalent:

(i) $T$ is power bounded.

(ii) $T$ and $T^{*}$ are mean square bounded on $B(X)$ and $B\left(X^{*}\right)$, respectively.

(iii) $T$ and $T^{*}$ are absolutely Cesàro bounded on $B(X)$ and $B\left(X^{*}\right)$, respectively.

Proof. The implications (i) $\Rightarrow$ (ii) $\Rightarrow$ (iii) are trivial.

Let us prove that (iii) $\Rightarrow$ (i). There exists $C>0$ such that for each $x \in X$ and $x^{*} \in X^{*}$ with $\|x\|=\left\|x^{*}\right\|=1$, and for all $N \in \mathbb{N}$, we have

$$
\frac{1}{N} \sum_{n=1}^{N}\left\|T^{n} x\right\| \leq C \text { and } \frac{1}{N} \sum_{n=1}^{N}\left\|T^{* n} x^{*}\right\| \leq C
$$

Suppose that $T$ is not power bounded, and fix any $K>4 C^{2}$. There exists $N$ such that $\left\|T^{N+1}\right\|>K$. Thus we find unit vectors $x \in X$ and $x^{*} \in X^{*}$ satisfying $\left|\left\langle T^{N+1} x, x^{*}\right\rangle\right|>K$. For each $n=1, \ldots, N$ we have

$$
K<\left|\left\langle T^{N+1} x, x^{*}\right\rangle\right|=\left|\left\langle T^{N+1-n} x, T^{* n} x^{*}\right\rangle\right| .
$$

So either $\left\|T^{N+1-n} x\right\| \geq \sqrt{K}$ or $\left\|T^{* n} x^{*}\right\| \geq \sqrt{K}$. Therefore,

$$
\frac{1}{N}\left(\sum_{n=1}^{N}\left\|T^{N+1-n} x\right\|+\sum_{n=1}^{N}\left\|T^{* n} x^{*}\right\|\right) \geq \sqrt{K}
$$


We then conclude

$$
\sqrt{K} \leq \frac{1}{N} \sum_{n=1}^{N}\left\|T^{n} x\right\|+\frac{1}{N} \sum_{n=1}^{N}\left\|T^{* n} x^{*}\right\| \leq 2 C<\sqrt{K}
$$

which is a contradiction.

Further consequences can be obtained for operators on Hilbert spaces.

Corollary 2.4. There exists a uniformly Kreiss bounded Hilbert space operator that is not absolutely Cesàro bounded.

Proof. Let $H$ be a separable infinite-dimensional Hilbert space with an orthonormal basis $\left(u_{k}\right)_{k \in \mathbb{N}}$. Let $0<\alpha<1 / 2$. Let $T \in B(H)$ be defined by $T u_{k}:=\left(\frac{k+1}{k}\right)^{\alpha} u_{k+1}$. A straightforward computation gives that $T$ is not absolutely Cesàro bounded since $\left\|T^{n} u_{1}\right\|=(n+1)^{\alpha} \rightarrow \infty$. Note that its adjoint $T^{*}$ is given by $T^{*} u_{k}=\left(\frac{k+1}{k}\right)^{\alpha} u_{k-1}$ for $k>1$ and $T^{*} u_{1}=0$. By Theorem 2.1, $T^{*}$ is absolutely Cesàro bounded, and hence uniformly Kreiss bounded. Since the uniform Kreiss boundedness is preserved by taking the adjoints, we deduce that $T$ is uniformly Kreiss bounded.

The following proposition complements Theorem 2.1, by showing that with $\alpha=$ $1 / p$ the theorem fails.

Proposition 2.1. Let $T$ be the weighted backward shift in $\ell^{p}(\mathbb{N})$ with $1 \leq p<\infty$ defined by $T e_{1}:=0, T e_{j}:=\left(\frac{j}{j-1}\right)^{1 / p} e_{j-1} \quad(j>1)$. Then $T$ is not Cesàro bounded.

Proof. Let $x_{n}:=\frac{1}{n^{1 / p}} \sum_{s=1}^{n} e_{s}$ with even $n$. It is clear that $\left\|x_{n}\right\|_{p}=1$. We have

$$
\begin{aligned}
\left\|\frac{1}{n} \sum_{j=0}^{n-1} T^{j} x_{n}\right\|_{p}^{p} & =\frac{1}{n^{p+1}}\left\|\sum_{j=0}^{n-1} \sum_{s=1}^{n} T^{j} e_{s}\right\|_{p}^{p}=\frac{1}{n^{p+1}}\left\|\sum_{s=1}^{n} e_{s} \sum_{j=s}^{n}\left(\frac{j}{s}\right)^{1 / p}\right\|_{p}^{p} \\
& =\frac{1}{n^{p+1}} \sum_{s=1}^{n}\left(\sum_{j=s}^{n}\left(\frac{j}{s}\right)^{1 / p}\right)^{p} \geq \frac{1}{n^{p+1}} \sum_{s=1}^{n / 2+1} \frac{1}{s}\left(\sum_{j=n / 2+1}^{n} j^{1 / p}\right)^{p}
\end{aligned}
$$

where

$$
\sum_{j=n / 2+1}^{n} j^{1 / p} \geq \int_{n / 2}^{n} t^{1 / p} d t \geq \frac{1}{p^{-1}+1}\left(n^{1+p^{-1}}-\left(\frac{n}{2}\right)^{1+p^{-1}}\right)=c n^{1+1 / p}
$$

with $c=\frac{p}{p+1}\left(1-\frac{1}{2^{1+p^{-1}}}\right)>0$. So

$$
\left\|n^{-1} \sum_{j=0}^{n-1} T^{j} x_{n}\right\|_{p}^{p} \geq \frac{1}{n^{p+1}} \sum_{s=1}^{n / 2} \frac{c^{p} n^{p+1}}{s} \geq c^{p} \ln \frac{n}{2} \rightarrow \infty
$$

as $n \rightarrow \infty$. Hence $T$ is not Cesàro bounded. 
Since we obviously have

$$
\frac{1}{n+1} T^{n+1}=I+(T-I) M_{n}(T)
$$

any Cesàro bounded operator satisfies $\left\|T^{n}\right\|=O(n)$. Moreover, Theorem 2.1 gives an example of a uniformly Kreiss bounded operator on $\ell^{1}(\mathbb{N})$ such that $\left\|T^{n}\right\|=(n+1)^{1-\varepsilon}$ with $0<\varepsilon<1$.

We concentrate now on Question 1.1 for operators on Hilbert spaces.

Theorem 2.3. Let $T$ be a uniformly Kreiss bounded operator on a Hilbert space $H$. Then $\lim _{n \rightarrow \infty} n^{-1}\left\|T^{n}\right\|=0$.

Proof. By [22, Corollary 3.2] there exists $C>0$ such that $\left\|\sum_{j=0}^{N-1}(\lambda T)^{j}\right\| \leq C N$ for all $\lambda,|\lambda|=1$ and all $N$. We need several claims.

Claim 1. Let $x \in H,\|x\|=1$ and $N \in \mathbb{N}$. Then

$$
\sum_{j=0}^{N-1}\left\|T^{j} x\right\|^{2} \leq C^{2} N^{2}
$$

Proof. Consider the normalized Lebesgue measure on the unit circle. We have

$$
\begin{gathered}
C^{2} N^{2} \geq \int_{|\lambda|=1}\left\|\left(I+\lambda T+\cdots+(\lambda T)^{N-1}\right) x\right\|^{2} d \lambda \\
=\sum_{j, k=0}^{N-1} \int_{|\lambda|=1}\left\langle(\lambda T)^{j} x,(\lambda T)^{k} x\right\rangle d \lambda=\sum_{j=0}^{N-1} \int_{|\lambda|=1}\left\langle(\lambda T)^{j} x,(\lambda T)^{j} x\right\rangle d \lambda=\sum_{j=0}^{N-1}\left\|T^{j} x\right\|^{2} .
\end{gathered}
$$

Claim 2. Let $0<M<N$ and $x \in H,\|x\|=1$. Then

$$
\sum_{j=0}^{M-1} \frac{\left\|T^{N} x\right\|^{2}}{\left\|T^{N-j} x\right\|^{2}} \leq C^{2} M^{2}
$$

Proof. Set $y=T^{N} x$. Since $T^{*}$ is also uniformly Kreiss bounded, we have by Claim 1

$$
\begin{gathered}
C^{2} M^{2}\|y\|^{2} \geq \sum_{j=0}^{M-1}\left\|T^{* j} y\right\|^{2} \\
\geq \sum_{j=0}^{M-1}\left|\left\langle T^{* j} y, \frac{T^{N-j} x}{\left\|T^{N-j} x\right\|}\right\rangle\right|^{2}=\sum_{j=0}^{M-1}\left|\left\langle y, \frac{T^{N} x}{\left\|T^{N-j} x\right\|}\right\rangle\right|^{2}=\|y\|^{2} \sum_{j=0}^{M-1} \frac{\left\|T^{N} x\right\|^{2}}{\left\|T^{N-j} x\right\|^{2}} .
\end{gathered}
$$

Hence

$$
\sum_{j=0}^{M-1} \frac{\left\|T^{N} x\right\|^{2}}{\left\|T^{N-j} x\right\|^{2}} \leq C^{2} M^{2}
$$


Claim 3. Let $x \in H,\|x\|=1$ and $N \in \mathbb{N}$. Then

$$
\sum_{j=0}^{N-1} \frac{1}{\left\|T^{j} x\right\|} \geq \frac{\sqrt{N}}{C}
$$

Proof. Let $a_{j}=\left\|T^{j} x\right\|$. By Claim $1, \sum_{j=0}^{N-1} a_{j}^{2} \leq C^{2} N^{2}$. So

$$
\sum_{j=1}^{N-1} a_{j} \leq\left(\sum_{j=0}^{N-1} a_{j}^{2}\right)^{1 / 2} \cdot \sqrt{N} \leq C N^{3 / 2}
$$

Let $B=N\left(\sum_{j=0}^{N-1} \frac{1}{a_{j}}\right)^{-1}$ and $A=N^{-1} \sum_{j=0}^{N-1} a_{j}$ be the harmonic and arithmetic means of $a_{j}$ 's for $j \in\{0, \ldots, N-1\}$, respectively. By the well-known inequality between these two means, we have

$$
\sum_{j=0}^{N-1} \frac{1}{\left\|T^{j} x\right\|}=\frac{N}{B} \geq \frac{N}{A}=N^{2}\left(\sum_{j=0}^{N-1} a_{j}\right)^{-1} \geq \frac{N^{2}}{C N^{3 / 2}}=\frac{\sqrt{N}}{C}
$$

Claim 4. Let $0<M_{1}<M_{2}<N$ and $\|x\|=1$. Then

$$
\sum_{j=M_{1}}^{M_{2}-1} \frac{\left\|T^{N-j} x\right\|^{2}}{\left\|T^{N} x\right\|^{2}} \geq \frac{\left(M_{2}-M_{1}\right)^{2}}{C^{2} M_{2}^{2}} .
$$

Proof. Let $a_{j}=\frac{\left\|T^{N-j} x\right\|^{2}}{\left\|T^{N} x\right\|^{2}}$. By Claim 2,

$$
\sum_{j=M_{1}}^{M_{2}-1} \frac{1}{a_{j}} \leq \sum_{j=0}^{M_{2}-1} \frac{1}{a_{j}} \leq C^{2} M_{2}^{2}
$$

Let $A$ and $B$ be the arithmetic and harmonic mean of $a_{j}$ 's for $j \in\left\{M_{1}, \ldots, M_{2}-1\right\}$, respectively. We have

$$
\sum_{j=M_{1}}^{M_{2}-1} a_{j}=\left(M_{2}-M_{1}\right) A \geq\left(M_{2}-M_{1}\right) B=\left(M_{2}-M_{1}\right)^{2}\left(\sum_{j=M_{1}}^{M_{2}-1} \frac{1}{a_{j}}\right)^{-1} \geq \frac{\left(M_{2}-M_{1}\right)^{2}}{C^{2} M_{2}^{2}} .
$$

Proof of Theorem 2.3. Suppose on the contrary that $\lim _{\sup _{n \rightarrow \infty}} n^{-1}\left\|T^{n}\right\|>c>0$.

Choose $K>8 C^{6} c^{-2}$. Find $N>2^{K+1}$ with $\left\|T^{N}\right\|>c N$ and $x \in H,\|x\|=1$ with

$$
\left\|T^{N} x\right\|>c N
$$

For $|\lambda|=1$ let $y_{\lambda}=\sum_{j=0}^{N-1} \frac{(\lambda T)^{j} x}{\left\|T^{j} x\right\|}$. Then

$$
\int_{|\lambda|=1}\left\|y_{\lambda}\right\|^{2} d \lambda=N
$$


and

$$
\int_{|\lambda|=1}\left\|\left(I+\lambda T+\cdots+(\lambda T)^{N-1}\right) y_{\lambda}\right\|^{2} d \lambda \leq C^{2} N^{2} \int_{|\lambda|=1}\left\|y_{\lambda}\right\|^{2} d \lambda=C^{2} N^{3} .
$$

On the other hand,

$$
\begin{gathered}
\int_{|\lambda|=1}\left\|\left(I+\lambda T+\cdots+(\lambda T)^{N-1}\right) y_{\lambda}\right\|^{2} d \lambda \\
=\int_{|\lambda|=1}\left\|\sum_{j=0}^{2 N-2}(\lambda T)^{j} x \sum_{r=0}^{\min \{N-1, j\}} \frac{1}{\left\|T^{r} x\right\|}\right\|^{2} d \lambda \\
=\sum_{j=0}^{2 N-2}\left\|T^{j} x\right\|^{2}\left(\sum_{r=0}^{\min \{N-1, j\}} \frac{1}{\left\|T^{r} x\right\|}\right)^{2} \geq \sum_{j=N-2^{K}}^{N}\left\|T^{j} x\right\|^{2}\left(\sum_{r=0}^{N-2^{K}} \frac{1}{\left\|T^{r} x\right\|}\right)^{2},
\end{gathered}
$$

where

$$
\sum_{r=0}^{N-2^{K}} \frac{1}{\left\|T^{r} x\right\|} \geq \frac{\sqrt{N-2^{K}}}{C} \geq \frac{\sqrt{N}}{C \sqrt{2}}
$$

and

$$
\sum_{j=N-2^{K}}^{N}\left\|T^{j} x\right\|^{2} \geq\left\|T^{N} x\right\|^{2} \sum_{k=0}^{K-1} \sum_{j=N-2^{k+1}}^{N-2^{k}-1} \frac{\left\|T^{j} x\right\|^{2}}{\left\|T^{N} x\right\|^{2}} \geq c^{2} N^{2} \sum_{k=0}^{K-1} \frac{2^{2 k}}{C^{2} 2^{2 k+2}}=\frac{c^{2} N^{2} K}{4 C^{2}} .
$$

Hence

$$
\int_{|\lambda|=1}\left\|\left(I+\lambda T+\cdots+(\lambda T)^{N-1}\right) y_{\lambda}\right\|^{2} d \lambda \geq \frac{c^{2} N^{2} K}{4 C^{2}} \cdot \frac{N}{2 C^{2}}=\frac{c^{2} K N^{3}}{8 C^{4}}>C^{2} N^{3},
$$

a contradiction. This finishes the proof.

Corollary 2.5. Any uniformly Kreiss bounded operator on a Hilbert space is mean ergodic.

Remark 2.2. By [10, Proposition 4] there exists a mean ergodic operator $T$ in a Hilbert space such that $\left\|T^{n}\right\| / n$ does not converges to 0 and thus it is not uniformly Kreiss bounded.

We are interested on the behavior of $\frac{\left\|T^{n}\right\|}{n}$ when $T$ is an absolutely Cesàro bounded operator. The following result provides an answer.

Theorem 2.4. Let $X$ be a Banach space, $C>0$ and let $T \in B(X)$ satisfy $\left\|T^{n}\right\| \leq$ $C n$ for all $n \in \mathbb{N}$. Then either $\lim _{n \rightarrow \infty} n^{-1}\left\|T^{n}\right\|=0$ or the set

$$
\left\{x \in X: \sup _{N} N^{-1} \sum_{n=1}^{N}\left\|T^{n} x\right\|=\infty\right\}
$$

is residual in $X$. 
Proof. Suppose that $\frac{\left\|T^{n}\right\|}{n} \not \rightarrow 0$. So there exists $c>0$ such that

$$
\limsup _{n \rightarrow \infty} n^{-1}\left\|T^{n}\right\|>c \text {. }
$$

For $s \in \mathbb{N}$ let

$$
M_{s}=\left\{x \in X: \sup _{N} N^{-1} \sum_{n=1}^{N}\left\|T^{n} x\right\|>s\right\} .
$$

Clearly $M_{s}$ is open.

We show first that each $M_{s}$ contains a unit vector. Let $s \in \mathbb{N}$. Find $N>$ $\exp \left(\frac{C s}{c}\right)+1$ with $\left\|T^{N}\right\|>c N$. Find a unit vector $x \in X$ such that $\left\|T^{N} x\right\|>c N$.

For $k=1, \ldots, N-1$ we have $\left\|T^{N} x\right\| \leq\left\|T^{k}\right\| \cdot\left\|T^{N-k} x\right\|$, and so

$$
\left\|T^{N-k} x\right\| \geq \frac{\left\|T^{N} x\right\|}{\left\|T^{k}\right\|} \geq \frac{c N}{C k} .
$$

Thus

$$
N^{-1} \sum_{j=1}^{N}\left\|T^{j} x\right\| \geq \sum_{k=1}^{N-1} \frac{c}{C k} \geq \frac{c}{C} \ln (N-1)>s
$$

and so $x \in M_{s}$.

We show that in fact each $M_{s}$ is dense. Fix $s \in \mathbb{N}, y \in X$ and $\varepsilon>0$. Let $s^{\prime}>\frac{s}{\varepsilon}$. Fix $x \in M_{s^{\prime}}$ with $\|x\|=1$. For each $j \in \mathbb{N}$ we have

$$
\left\|T^{j}(y+\varepsilon x)\right\|+\left\|T^{j}(y-\varepsilon x)\right\| \geq 2 \varepsilon\left\|T^{j} x\right\| .
$$

So

$\sup _{N} N^{-1} \sum_{j=1}^{N}\left\|T^{j}(y+\varepsilon x)\right\|+\sup _{N} N^{-1} \sum_{j=1}^{N}\left\|T^{j}(y-\varepsilon x)\right\| \geq \sup _{N} \frac{2 \varepsilon}{N} \sum_{j=1}^{N}\left\|T^{j} x\right\|>2 \varepsilon s^{\prime}>2 s$.

Hence either $y+\varepsilon x \in M_{s}$ or $y-\varepsilon x \in M_{s}$. Since $\varepsilon>0$ was arbitrary, $M_{s}$ is dense.

By the Baire category theorem,

$$
\bigcap_{s+1}^{\infty} M_{s}=\left\{x \in X: \sup _{N} N^{-1} \sum_{j=1}^{N}\left\|T^{j} x\right\|=\infty\right\}
$$

is a residual set.

The following example is due to Assani. See [3, page 938], [12, page 10] and [2, Theorem 5.4] for more details.

Example 2.2. Let $H$ be $\mathbb{R}^{2}$ or $\mathbb{C}^{2}$ and $T=\left(\begin{array}{cc}-1 & 2 \\ 0 & -1\end{array}\right)$. It is clear that

$$
T^{n}=\left(\begin{array}{cc}
(-1)^{n} & (-1)^{n-1} 2 n \\
0 & (-1)^{n}
\end{array}\right)
$$

and $\sup _{n \in \mathbb{N}}\left\|M_{n}(T)\right\|<\infty$. Then $T$ is Cesàro bounded and $\frac{\left\|T^{n} x\right\|}{n}$ does not converge to 0 for some $x \in H$. Hence $T$ is not mean ergodic. 
Since Cesàro bounded operators $T$ satisfy $\left\|T^{n}\right\|=O(n)$, by Theorem 2.4 we immediately obtain the following result.

Corollary 2.6. Let $T \in B(X)$ be an absolutely Cesàro bounded operator. Then $\lim _{n \rightarrow \infty} \frac{\left\|T^{n}\right\|}{n}=0$.

As consequence, we obtain a result that, for operators on Banach spaces, slightly improves Lorch theorem [1].

Corollary 2.7. Any absolutely Cesàro bounded operator on a reflexive Banach space is mean ergodic.

Hence by Corollary 2.3, we can also provide more examples of mean ergodic and mixing operators on $\ell^{p}(\mathbb{N})$ for $1<p<\infty$.

It is worth to mention that results of this type already appear in the $\mathrm{PhD}$ Thesis of Beltrán Meneu [4], provided by the fourth author (see Section 3.7 in [4]), and in [2].

For $0<\varepsilon<1$, by Theorem 2.1 we have examples of absolutely Cesàro bounded operators on $\ell^{2}(\mathbb{N})$ such that $\left\|T^{n}\right\|=(n+1)^{\frac{1}{2}-\varepsilon}$. On the other hand, if there exists $\varepsilon>0$ such that $\left\|T^{n}\right\| \geq C n^{\frac{1}{2}+\varepsilon}$ for all $n$ in a Hilbert space, then by [23, Theorem 3], there exists $x \in X$ such that $\left\|T^{n} x\right\| \rightarrow \infty$, thus $T$ is not absolutely Cesàro bounded. Hence it is natural to ask: does every absolutely Cesàro bounded operator on a Hilbert space satisfy $\lim _{n \rightarrow \infty} n^{-1 / 2}\left\|T^{n}\right\|=0$ ?

Theorem 2.5. Let $H$ be a Hilbert space and let $T \in B(H)$ be an absolutely Cesàro bounded operator. Then $\lim _{n \rightarrow \infty} \frac{\left\|T^{n}\right\|}{n^{1 / 2}}=0$.

Proof. By [22, corollary 3.2] there exists $C>0$ such that $N^{-1} \sum_{n=0}^{N-1}\left\|T^{n} x\right\|<C\|x\|$ for all $N \in \mathbb{N}$ and $x \in H$.

Suppose on the contrary that $\limsup _{n \rightarrow \infty} N^{-1 / 2}\left\|T^{n}\right\|>0$. We distinguish two cases:

Case I. Suppose that $\lim \sup _{n \rightarrow \infty} n^{-1 / 2}\left\|T^{n}\right\|=\infty$.

Then there exist positive integers $N_{1}<N_{2}<\cdots$ and positive constants $K_{1}<$ $K_{2}<\cdots$ with $\lim _{m \rightarrow \infty} K_{m}=\infty$ such that $\left\|T^{N_{m}}\right\|>K_{m} N_{m}^{1 / 2}$ and

$$
\left\|T^{j}\right\| \leq 2 K_{m} j^{1 / 2} \quad\left(j \leq N_{m}\right) .
$$

Let $x_{m} \in H$ be a unit vector satisfying $\left\|T^{N_{m}} x_{m}\right\|>K_{m} N_{m}^{1 / 2}$.

Let $N_{m}^{\prime}=\left[\frac{N_{m}}{6}\right] \quad$ (the integer part). Consider the set

$$
\left\{\left\|T^{j} x_{m}\right\|: 2 N_{m}^{\prime} \leq j<4 N_{m}^{\prime}\right\} .
$$

Let $A_{m}$ be the median of this set. More precisely, we have

$$
\operatorname{card}\left\{j: 2 N_{m}^{\prime} \leq j<4 N_{m}^{\prime},\left\|T^{j} x_{m}\right\| \geq A_{m}\right\} \geq N_{m}^{\prime} \quad \text { and }
$$




$$
\operatorname{card}\left\{j: 2 N_{m}^{\prime} \leq j<4 N_{m}^{\prime},\left\|T^{j} x_{m}\right\| \leq A_{m}\right\} \geq N_{m}^{\prime}
$$

We have

$$
4 N_{m}^{\prime} C \geq \sum_{j=0}^{4 N_{m}^{\prime}-1}\left\|T^{j} x_{m}\right\| \geq \sum_{j=2 N_{m}^{\prime}}^{4 N_{m}^{\prime}-1}\left\|T^{j} x_{m}\right\| \geq N_{m}^{\prime} A_{m}
$$

So $A_{m} \leq 4 C$ (note that this estimate does not depend on $m$ ).

For $\lambda \in \mathbb{C},|\lambda|=1$ let

$$
y_{m, \lambda}=\sum_{j=1}^{N_{m}} \frac{(\lambda T)^{j} x_{m}}{\left\|T^{j} x_{m}\right\|}
$$

Then

$$
\begin{gathered}
\int\left\|y_{m, \lambda}\right\|^{2} d \lambda=\int \sum_{j, j^{\prime}=1}^{N_{m}} \frac{\left\langle\lambda^{j} T^{j} x_{m}, \lambda^{j^{\prime}} T^{j^{\prime}} x_{m}\right\rangle}{\left\|T^{j} x_{m}\right\| \cdot\left\|T^{j^{\prime}} x_{m}\right\|} d \lambda \\
=\int \sum_{j=1}^{N_{m}} \frac{\left\langle T^{j} x_{m}, T^{j} x_{m}\right\rangle}{\left\|T^{j} x_{m}\right\|^{2}} d \lambda=N_{m} .
\end{gathered}
$$

Let

$$
u_{m, \lambda}=\left(I+\lambda T+\cdots+(\lambda T)^{N_{m}-1}\right) y_{m, \lambda} .
$$

Then $\left\|u_{m, \lambda}\right\| \leq C N_{m}\left\|y_{m, \lambda}\right\|$ and

$$
\int\left\|u_{m, \lambda}\right\|^{2} d \lambda \leq C^{2} N_{m}^{2} \int\left\|y_{m, \lambda}\right\|^{2} d \lambda=C^{2} N_{m}^{3}
$$

On the other hand,

$$
u_{m, \lambda}=\sum_{j=1}^{N_{m}}(\lambda T)^{j} x_{m} \sum_{k=1}^{j} \frac{1}{\left\|T^{k} x_{m}\right\|}+\sum_{j=N_{m}+1}^{2 N_{m}-1}(\lambda T)^{j} x_{m} \sum_{k=j-N_{m}+1}^{N_{m}} \frac{1}{\left\|T^{k} x_{m}\right\|} .
$$

As above,

$$
\begin{gathered}
\int\left\|u_{m, \lambda}\right\|^{2} d \lambda \geq \sum_{j=1}^{N_{m}}\left\|T^{j} x_{m}\right\|^{2}\left(\sum_{k=1}^{j} \frac{1}{\left\|T^{k} x_{m}\right\|}\right)^{2} \geq\left\|T^{N_{m}} x_{m}\right\|^{2}\left(\sum_{k=2 N_{m}^{\prime}}^{4 N_{m}^{\prime}-1} \frac{1}{\left\|T^{k} x_{m}\right\|}\right)^{2} \\
\geq K_{m}^{2} N_{m} \cdot\left(\frac{N_{m}^{\prime}}{A_{m}}\right)^{2} \geq K_{m}^{2} \cdot \text { const } \cdot N_{m}^{3} .
\end{gathered}
$$

Since $K_{m} \rightarrow \infty$, this is a contradiction.

Case $I I$. Let $K$ satisfy $0<K<\lim \sup _{n \rightarrow \infty} n^{-1 / 2}\left\|T^{n}\right\|<2 K$.

Let $N_{0}$ satisfy $n^{-1 / 2}\left\|T^{n}\right\| \leq 2 K \quad\left(n \geq N_{0}\right)$. Find an increasing sequence $\left(N_{m}\right)$ of positive integers such that $\left\|T^{N_{m}}\right\|>K N_{m}^{1 / 2}$. Find $x_{m},\left\|x_{m}\right\|=1$ such that $\left\|T^{N_{m}} x_{m}\right\|>K N_{m}^{1 / 2}$.

As in case I, let $N_{m}^{\prime}=\left[\frac{N_{m}}{6}\right]$ and let $A_{m}$ be the median of the set

$$
\left\{\left\|T^{j} x_{m}\right\|: 2 N_{m}^{\prime} \leq j<4 N_{m}^{\prime}\right\} \text {. }
$$


Again one has $A_{m} \leq 4 C$.

As in case I, for $|\lambda|=1$ let

$$
y_{m, \lambda}=\sum_{j=1}^{N_{m}} \frac{(\lambda T)^{j} x_{m}}{\left\|T^{j} x_{m}\right\|}
$$

and

$$
u_{m, \lambda}=\left(I+\lambda T+\cdots+(\lambda T)^{N_{m}-1}\right) y_{m, \lambda} .
$$

Again we have $\int\left\|y_{m, \lambda}\right\|^{2} d \lambda=N_{m}$ and

$$
\int\left\|u_{m, \lambda}\right\|^{2} d \lambda \leq C^{2} N_{m}^{3}
$$

On the other hand,

$$
u_{m, \lambda}=\sum_{j=1}^{N_{m}}(\lambda T)^{j} x_{m} \sum_{k=1}^{j} \frac{1}{\left\|T^{k} x_{m}\right\|}+\sum_{j=N_{m}+1}^{2 N_{m}-1}(\lambda T)^{j} x_{m} \sum_{k=j-N_{m}+1}^{N_{m}} \frac{1}{\left\|T^{k} x_{m}\right\|}
$$

and

$$
\begin{gathered}
\int\left\|u_{m, \lambda}\right\|^{2} d \lambda \geq \sum_{j=1}^{N_{m}}\left\|T^{j} x_{m}\right\|^{2}\left(\sum_{k=1}^{j} \frac{1}{\left\|T^{k} x_{m}\right\|}\right)^{2} \geq \sum_{j=4 N_{m}^{\prime}}^{N_{m}-1}\left\|T^{j} x_{m}\right\|^{2}\left(\sum_{k=2 N_{m}^{\prime}}^{4 N_{m}^{\prime}-1} \frac{1}{\left\|T^{k} x_{m}\right\|}\right)^{2} \\
\geq \sum_{j=4 N_{m}^{\prime}}^{N_{m}-1}\left\|T^{j} x_{m}\right\|^{2}\left(\frac{N_{m}^{\prime}}{A_{m}}\right)^{2} .
\end{gathered}
$$

Moreover, for $4 N_{m}^{\prime} \leq j<N_{m}$ we have

$$
K N_{m}^{1 / 2}<\left\|T^{N_{m}} x_{m}\right\| \leq\left\|T^{N_{m}-j}\right\| \cdot\left\|T^{j} x_{m}\right\| \leq 2 K\left(N_{m}-j\right)^{1 / 2}\left\|T^{j} x_{m}\right\| .
$$

So

$$
\sum_{j=4 N_{m}^{\prime}}^{N_{m}}\left\|T^{j} x_{m}\right\|^{2} \geq \sum_{j=4 N_{m}^{\prime}}^{N_{m}-1} \frac{N_{m}}{4\left(N_{m}-j\right)} \geq \frac{N_{m}}{4} \sum_{j=1}^{2 N_{m}^{\prime}} \frac{1}{j} \geq \frac{N_{m} \ln \left(2 N_{m}^{\prime}\right)}{4} .
$$

Hence

$$
\int\left\|u_{m, \lambda}\right\|^{2} d \lambda \geq \text { const } \cdot N_{m}^{3} \ln \left(2 N_{m}^{\prime}\right)
$$

a contradiction.

Figure 2 summarizes the implications between the properties studied here and the behaviour of $\left\|T^{n}\right\|$. 
Uniformly Kreiss bounded $\longleftarrow$ absolutely Cesàro bounded

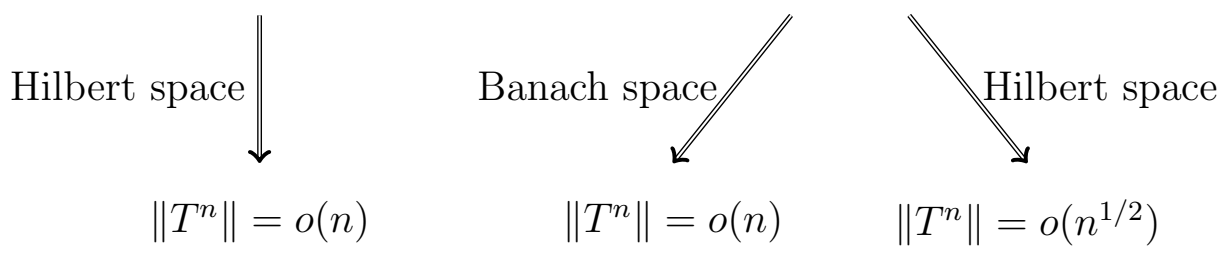

Figure 2: Behaviour of $\left\|T^{n}\right\|$ for uniformly Kreiss and Cesàro bounded operators.

We finish the paper with some questions.

Question 2.1. Are there absolutely Cesàro bounded operators on Hilbert spaces which are not strongly Kreiss bounded? (Corollary 2.2 gives examples only in $\ell^{p}(\mathbb{N})$ with $1<p<2)$.

Question 2.2. Can we find strongly Kreiss bounded operators which are not absolutely Cesàro bounded?

Question 2.3. Is there any absolutely Cesàro bounded operator $T$ on a Hilbert space which is not mean square bounded?

\section{Acknowledgements}

We are extremely indebted to the referee whose valuable suggestions and advice, together with several references, produced an improvement of this paper.

\section{References}

[1] A. Albanese, J. Bonet and W.J. Ricker, Mean ergodic operators in Fréchet spaces, Ann. Acad. Sci. Fenn. Math., 34 (2009), 401-436.

[2] A. Aleman and L. Suciu, On ergodic operator means in Banach spaces, Integral Equations Operator Theory, 85 (2016), 259-287.

[3] I. Assani, Sur les opérateurs à puissances bornées et le théorème ergodique ponctuel dans $L^{p}[0,1], 1<p<\infty$, Canad. J. Math., 38 (1986), no. 4, 937-946.

[4] M.J. Beltrán-Meneu, Operators on weighted spaces of holomorphic functions, PhD Thesis, Universitat Politècnica de València, 2014.

[5] M. J. Beltrán, J. Bonet, C. Fernández, Classical operators on weighted Banach spaces of entire functions, Proc. Amer. Math. Soc. 141 (2013), 4293-4303.

[6] M. J. Beltrán, M. C. Gómez-Collado, E. Jordá, D. Jornet, Mean ergodic composition operators on Banach spaces of holomorphic functions, J. Funct. Anal. 270 (2016), 4369-4385. 
[7] N. C. Bernardes Jr, A. Bonilla, V. Müller and A. Peris, Distributional chaos for linear operators, J. Funct. Anal., 265 (2013), 2143-2163.

[8] N. C. Bernardes Jr, A. Bonilla, A. Peris and X. Wu, Distributional chaos for operators in Banach spaces, J. Math. Anal. Appl., 459 (2018), 797-821.

[9] J. Bonet, Dynamics of the differentiation operator on weighted spaces of entire functions, Math. Z. 261 (2009), 649-657.

[10] Y. Derriennic, On the mean ergodic theorem for Cesàro bounded operators, Colloq. Math., 84/85 (2000), part 2, 443-455.

[11] Y. Derriennic and M. Lin, On invariant measures and ergodic theorems for positive operators, J. Funct. Anal., 13 (1973), 252-267.

[12] R. Émilion, Mean-bounded operators and mean ergodic theorems, J. Funct. Anal., 61 (1985), no. 1, 1-14.

[13] A. Gomilko and J. Zemánek, On the uniform Kreiss resolvent condition, (Russian) Funktsional. Anal. i Prilozhen., 42 (2008), no. 3, 81-84; translation in Funct. Anal. Appl. 42 (2008), no. 3, 230-233.

[14] K.-G. Grosse-Erdmann and A. Peris, Linear Chaos, Springer, London, 2011.

[15] B. Hou and L. Luo, Some remarks on distributional chaos for bounded linear operators, Turk. J. Math., 39 (2015), 251-258.

[16] E. Hille, Remarks on ergodic theorems, Trans. Amer. Math. Soc., 57, (1945). 246-269.

[17] I. Kornfeld and W. Kosek, Positive $L^{1}$ operators associated with nonsingular mappings and an example of E. Hille, Colloq. Math., 98 (2003), no. 1, 63-77.

[18] W. Kosek, Example of a mean ergodic $L^{1}$ operator with the linear rate of growth, Colloq. Math. 124 (2011), no. 1, 15-22.

[19] U. Krengel, Ergodic theorems. De Gruyter Studies in Mathematics, 6. Walter de Gruyter and Co., Berlin, 1985.

[20] C. Lubich and O. Nevanlinna, On resolvent conditions and stability estimates, BIT, 31 (1991), no. 2, 293-313.

[21] C.A. McCarthy, A strong resolvent condition does not imply powerboundedness, Chalmers Institute of Technology and the University of Göteborg, Preprint No 15 (1971).

[22] A. Montes-Rodríguez, J. Sánchez-Álvarez and J. Zemánek, Uniform Abel-Kreiss boundedness and the extremal behavior of the Volterra operator, Proc. London Math. Soc., 91 (2005),761-788. 
[23] V. Müller and J. Vrsovsky, Orbits of linear operators tending to infinity, Rocky Mountain J. Math., 39 (2009), 219-230.

[24] O. Nevanlinna, Resolvent conditions and powers of operators, Studia Math., 145 (2001), no. 2, 113-134.

[25] J. Neveu, Mathematical foundations of the calculus of probability. Translated by Amiel Feinstein Holden-Day, Inc., San Francisco, Calif.-London-Amsterdam 1965

[26] A. L. Shields, On Möbius Bounded operators, Acta Sci. Math., (Szeged), 40 (1978), 371-374.

[27] J. C. Strikwerda and B. A. Wade, A survey of the Kreiss matrix theorem for power bounded families of matrices and its extensions, Linear operators (Warsaw, 1994), 339-360, Banach Center Publ., 38, Polish Acad. Sci., Warsaw, 1997.

[28] L. Suciu, Ergodic behaviors of the regular operator means, Banach J. Math. Anal., 11 (2017), no. 2, 239-265.

[29] L. Suciu and J. Zemánek, Growth conditions on Cesàro means of higher order, Acta Sci. Math (Szeged), 79 (2013), 545-581.

[30] Y. Tomilov and J. Zemánek, A new way of constructing examples in operator ergodic theory, Math. Proc. Cambridge Philos. Soc., 137 (2004), no. 1, 209-225.

[31] J. A. Van Casteren, Boundedness properties of resolvents and semigroups of operators. Linear operators (Warsaw, 1994), 59-74, Banach Center Publ., 38, Polish Acad. Sci. Inst. Math., Warsaw, 1997.

T. Bermúdez

Departamento de Análisis Matemático, Universidad de la Laguna, 38271, La Laguna

(Tenerife), Spain.

e-mail: tbermude@ull.es

A. Bonilla

Departamento de Análisis Matemático, Universidad de la Laguna, 38271, La Laguna

(Tenerife), Spain.

e-mail: abonilla@ull.es

V. Müller

Mathematical Institute, Czech Academy of Sciences, Zitná 25, 11567 Prague 1,

Czech Republic.

e-mail: muller@math.cas.cz

A. Peris

Institut Universitari de Matemàtica Pura i Aplicada, Universitat Politècnica de València, Edifici $8 E$, Acces F, 4a planta, 46022 València, Spain.

e-mail: aperis@mat.upv.es 